06

\title{
Генерация второй гармоники монослоем сферических двухслойных наночастиц
}

\author{
(C) Т.М. Чмерева, М.Г. Кучеренко, Ф.Ю. Мушин, В.М. Налбандян \\ Оренбургский государственный университет, \\ 460018 Оренбург, Россия \\ e-mail: chmereva@yandex.ru
}

Поступила в редакцию 03.01.2021 г.

В окончательной редакции 03.01.2021 г.

Принята к публикации 27.04.2021 г.

\begin{abstract}
Предложена модель расчета интенсивности второй гармоники (ВГ) при отражении света от монослоя состоящих из диэлектрического ядра и плазмонной оболочки сферических наночастиц, расположенного вблизи границы раздела двух оптически прозрачных сред. Рассчитана создаваемая наночастицами нелинейная поляризация границы раздела, являющаяся источником ВГ. Обнаружено увеличение интенсивности отраженной ВГ, вызванное дипольным и квадрупольным плазмонными резонансами в наночастицах. Показано, что спектральные положения максимумов интенсивности ВГ существенно зависят от размера ядра частицы и его диэлектрической проницаемости.
\end{abstract}

Ключевые слова: двухслойная наночастица, плазмонный резонанс, нелинейная поверхностная поляризация, генерация второй гармоники.

DOI: $10.21883 /$ OS.2021.08.51201.1946-21

\section{Введение}

Отличительной особенностью современных исследований в области оптики является повышенный интерес к композитным наноструктурам, содержащим плазмонные наночастицы. Уникальные оптические свойства металлических наночастиц используются при разработке сенсоров и датчиков, нанолазеров, оптических наноантенн, органических солнечных элементов. Один из интересных и важных с прикладной точки зрения эффектов - это генерация оптических гармоник при взаимодействии лазерного излучения с металлическими поверхностями и наноструктурами. В последнее время нелинейные свойства металлических и полупроводниковых наночастиц и композитных структур на их основе активно исследуются как экспериментально [1-5], так и теоретически [6-11].

Генерация второй гармоники (ГВГ) сферическими наночастицами золота размером $150 \mathrm{~nm}$ изучалась в работе [1]. Путем сравнения экспериментальных результатов с теоретическими моделями был оценен вклад различных нелинейных источников, а также показано, что интерференционные эффекты между дипольными и октупольными плазмонами могут быть использованы в качестве критериев для идентификации локального поверхностного и нелокального объемного вкладов в ГВГ. О ГВГ путем облучения монослоев наночастиц $\mathrm{Ga}$ высокой чистоты, встроенных в матрицу $\mathrm{SiOx}$, фемтосекундными лазерными импульсами на длине волны $800 \mathrm{~nm}$ сообщается в работе [2]. Авторы наблюдали заметное усиление ГВГ, которое связывали с плавлением. Эксперименты, направленные на выявление возможных механизмов ГВГ в литографически подготовленных массивах металлических наночастиц, описаны в работе [3]. В этой работе предлагается покрывать массивы тем же материалом, на котором они созданы, создавая таким образом центросимметричную среду, в которой ГВГ может быть вызвана не асимметрией в среде, а нелокальными или запаздывающими механизмами в частицах. Путем настройки резонансов наночастиц на длину волны возбуждения сигнал второй гармоники (ВГ) может быть существенно усилен. В работах $[4,5]$ рассматриваются массивы наночастиц золота, состоящие из нецентросимметричных (активных) частиц с нелинейно-оптическим откликом второго порядка. В [4] показано, что добавление в массив центросимметричных (пассивных) частиц приводит к усилению ГВГ от активных частиц. В [5] обнаружено существенное усиление сигнала ГВГ при уменьшении поверхностной плотности активных частиц в массиве. По мнению авторов полученные ими результаты дают совершенно новую концепцию оптимизации нелинейных откликов метаматериалов.

Наиболее строгий подход к описанию генерации оптических гармоник сферической наночастицей в поле плоской волны основан на решении задачи Ми о рассеянии $[6,7]$. Он позволяет учесть рассеяние падающих и генерируемых волн, однако является достаточно сложным. В работах [8-10] показано, что учет неоднородности электрического поля монохроматической волны в пределах сферической наночастицы позволяет определить дипольный и квадрупольный моменты, которые порождают излучение на частоте ВГ. Нелинейный дипольный момент направлен вдоль волнового вектора падающей волны, а тензор квадрупольного момента соответствует симметричному относительно направления вектора напряженности электрического поля квадруполю. При вы- 
полнении условий возникновения локализованных плазмонных резонансов в наночастице наблюдается резкое возрастание интенсивности ВГ. Для оценки излучения ВГ, генерируемого металлическими наночастицами произвольной формы, в работах $[11,12]$ используется система поверхностных интегральных уравнений, которая решается с помощью процедуры тестирования Галеркина и базисных функций Рао-Уилтона-Гиллсона. Правильность предложенного авторами подхода подтверждается сравнениями с аналитическим решением Ми.

В настоящей работе теоретически изучена ГВГ неупорядоченным монослоем сферических наночастиц со структурой „ядро-оболочка“, расположенным на границе двух диэлектриков. В поле электромагнитной волны наночастицы приобретают дипольный и квадрупольный моменты, являющиеся источниками ВГ, и в случае наночастиц малого по сравнению с длиной волны диаметра монослой может рассматриваться как бесконечно тонкий лист на границе раздела диэлектрических сред, обладающий нелинейной поверхностной поляризацией. Нелинейная поляризация границы раздела была вычислена в рамках подхода, предложенного в $[13,14]$ для слоя жидкого кристалла и использующего нелинейные поляризуемости отдельной молекулы. Для нахождения нелинейных дипольной и квадрупольной поляризуемостей наночастицы использовался метод, основанный на учете неоднородности электромагнитного поля плоской волны в пределах наночастицы и вблизи нее [9]. Амплитуда отраженной ВГ определялась из граничных условий, учитывающих наличие нелинейной поверхностной поляризации, для тангенциальных компонент напряженностей электрического и магнитного полей удвоенной частоты [15].

\section{Нелинейный отклик сферической двухслойной наночастицы}

На рис. 1 изображен монослой плазмонных наночастиц с диэлектрическим ядром и металлической оболочкой, расположенный вблизи плоской границы двух оптически прозрачных сред с диэлектрическими проницаемостями $\varepsilon_{1}$ и $\varepsilon_{2}$. При падении на границу раздела плоской волны наночастицы оказываются в поле преломленной волны. Монослой предполагается неупорядоченным, чтобы исключить возникновение коллективных плазмонных мод. Кроме того, также исключается влияние частиц друг на друга.

Как известно $[1,16]$, нелинейный оптический отклик наночастиц из изотропных и центросимметричных материалов, к которым относятся благородные металлы, феноменологически описывается нелинейной поляризацией, имеющей локальный поверхностный и нелокальный объемный вклады. Вектор поверхностной поляризации определяется как

$$
\mathbf{P}_{s}^{2 \omega}(\mathbf{r})= \pm \chi_{s}^{(2)}: \mathbf{E}^{\omega}(\mathbf{r}) \mathbf{E}^{\omega}(\mathbf{r})
$$

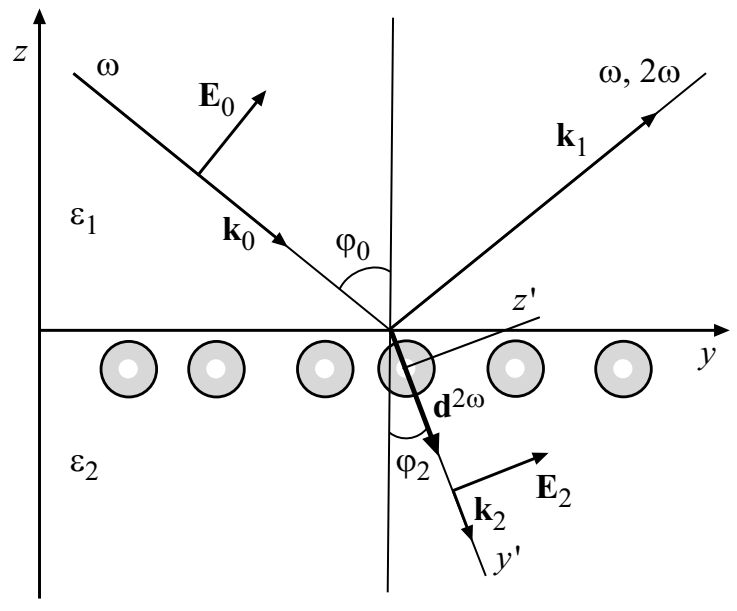

Рис. 1. Падение $p$-поляризованной волны на поверхность среды с монослоем наночастиц.

где $\chi_{s}^{(2)}-$ тензор поверхностной восприимчивости второго порядка, $\mathbf{E}^{\omega}(\mathbf{r})$ - напряженность поля основной частоты внутри оболочки частицы. Верхний знак относится к внешней поверхности оболочки наночастицы, нижний знак - к внутренней.

Тензор поверхностной восприимчивости второго порядка $\chi_{s}^{(2)}$ имеет три независимых элемента: $\chi_{\perp \perp \perp}$, $\chi_{\perp\|\|}$ и $\chi_{\|\perp\|}=\chi_{\|\| \perp}$, где индексы $\perp$ и $\|$ обозначают перпендикулярное и касательное направления к поверхности соответственно. Как отмечается в работах $[1,17]$, элемент $\chi_{\perp \perp \perp}$ вносит наибольший вклад в ГВГ наночастицей из благородного металла, а вклад $\chi_{\perp\|\|}$ пренебрежимо мал.

Вектор объемной поляризации определяется выражением [1]

$$
\mathbf{P}_{b}^{2 \omega}(\mathbf{r})=\chi_{b}^{(2)} \nabla\left(\mathbf{E}^{\omega}(\mathbf{r}) \mathbf{E}^{\omega}(\mathbf{r})\right)
$$

где $\chi_{b}^{(2)}-$ нелинейная объемная восприимчивость второго порядка.

Вектор объемной нелинейной поляризации задает нелинейный объемный ток $\mathbf{j}^{(2 \omega)}=\partial \mathbf{P}_{b}^{2 \omega} / \partial t$, который уравнением неразрывности связан с нелинейной объемной плотностью заряда. Как следует из работы [9], для однородной сферической частицы эта плотность заряда равна нулю. В области оболочки объемная плотность заряда хотя и отлична от нуля, но достаточно мала, и ее вкладом в ГВГ можно пренебречь. Поэтому процесс ГВГ будет обусловлен в основном поверхностными поляризациями оболочки наночастицы (1) и нормальными составляющими вектора объемной поляризации оболочки (2) на ее границах.

Касательная составляющая вектора поверхностной поляризации

$$
\mathbf{P}_{s \|}^{2 \omega}\left(R_{1(2)}\right)= \pm\left. 2 \chi_{\|\perp\|} E_{\perp}^{\omega}(\mathbf{r}) \mathbf{E}_{\|}^{\omega}(\mathbf{r})\right|_{r=R_{1(2)}}
$$


и нормальная составляющая вектора объемной поляризации

$$
\mathbf{P}_{b \perp}^{2 \omega}\left(R_{1(2)}\right)= \pm\left.\chi_{b}^{(2)} \nabla_{r}\left(\mathbf{E}^{\omega}(\mathbf{r}) \mathbf{E}^{\omega}(\mathbf{r})\right)\right|_{r=R_{1(2)}},
$$

вычисленная на границах оболочки, определяют нелинейную поверхностную плотность заряда [8]

$$
\sigma_{s}^{2 \omega}\left(R_{1(2)}\right)=-\nabla_{\|} \cdot \mathbf{P}_{s \|}^{2 \omega}\left(R_{1(2)}\right)+\mathbf{P}_{b \perp}^{2 \omega}\left(R_{1(2)}\right),
$$

где $\nabla_{\|}$- оператор градиента вдоль поверхности.

Нормальная к поверхностям сферической оболочки составляющая вектора поверхностной поляризации определяется квадратом радиальной составляющей $\left(E_{r}^{\omega}(\mathbf{r})=E_{\perp}^{\omega}(\mathbf{r})\right)$ электрического поля внутри оболочки:

$$
P_{s \perp}^{2 \omega}\left(R_{1(2)}\right)= \pm\left.\chi_{\perp \perp \perp} E_{\perp}^{\omega}(\mathbf{r}) E_{\perp}^{\omega}(\mathbf{r})\right|_{r=R_{1(2)}} .
$$

В квазистатическом приближении неоднородность в пределах частицы поля падающей плоской волны можно учесть, сохранив в разложении экспоненты линейное по волновому числу слагаемое [9] $E_{2} e^{i k_{2} y^{\prime}} \mathbf{e}_{z^{\prime}}=\left(1+i k_{2} y^{\prime}\right) E_{2} \mathbf{e}_{z^{\prime}}$, где $E_{2}-$ амплитуда преломленной волны, $\mathbf{z}_{z^{\prime}}$ - орт оси $z^{\prime}$ системы координат $x^{\prime} y^{\prime} z^{\prime}$, связанной с наночастицей, как показано на рис. 1. При этом напряженность результирующего поля основной частоты $\mathbf{E}^{\omega}(\mathbf{r})$ в присутствии наночастицы удобно искать как сумму напряженностей поля падающей волны и поля, рассеянного частицей [9]. Тогда потенциал поля, рассеянного частицей, будет являться решением уравнения Лапласа. Результирующее поле должно удовлетворять условиям непрерывности касательной составляющей напряженности электрического поля и нормальной составляющей вектора электрической индукции на внутренней и внешней поверхностях оболочки частицы.

Нелинейные поверхностные плотности заряда (3) на поверхностях оболочки наночастицы, вычисленные с результирующим полем $\mathbf{E}^{\omega}(\mathbf{r})$ внутри оболочки, содержат слагаемые с разными угловыми зависимостями:

$$
\begin{aligned}
& \sigma_{s}^{2 \omega}\left(R_{1(2)}\right)=F_{0}\left(R_{1(2)}\right)\left(3 \cos ^{2} \theta-1\right)+F_{1}\left(R_{1(2)}\right) \sin \theta \sin \varphi \\
& +F_{2}\left(R_{1(2)}\right) \sin ^{3} \theta \sin \varphi+F_{3}\left(R_{1(2)}\right)
\end{aligned}
$$

где $\theta$ и $\varphi-$ полярный и азимутальный углы в системе координат $x^{\prime} y^{\prime} z^{\prime}$, связанной с частицей, ось $z^{\prime}$ которой сонаправлена с вектором напряженности поля преломленной волны $\mathbf{E}_{2}$ (рис. 1). Для коэффициентов $F_{0}, F_{1}, F_{2}$ получаются следующие выражения:

$$
\begin{aligned}
& F_{0}\left(R_{1(2)}\right)= \pm \frac{2 \chi_{\|\perp\|}}{R_{1(2)}}\left(E_{2}-B_{0}+\frac{2 C_{0}}{R_{1(2)}^{3}}\right) \\
& \times\left(-E_{2}+B_{0}+\frac{C_{0}}{R_{1(2)}^{3}}\right) \pm 6 \chi_{b}^{(2)}\left(\frac{\left(E_{2}-B_{0}\right) C_{0}}{R_{1(2)}^{4}}+\frac{C_{0}^{2}}{R_{1(2)}^{7}}\right),
\end{aligned}
$$

$$
\begin{gathered}
F_{1}\left(R_{1(2)}\right)= \pm 2 \chi_{\|\perp\|}\left[( E _ { 2 } - B _ { 0 } ) \left(-6 i k_{2} E_{2}+13 B_{1}\right.\right. \\
\left.\left.-\frac{2 C_{1}}{R_{1(2)}^{5}}\right)+\frac{C_{0}}{R_{1(2)}^{3}}\left(-3 i k_{2} E_{2}+8 B_{1}+\frac{23 C_{1}}{R_{1(2)}^{5}}\right)\right] \\
\pm 2 \chi_{b}^{(2)}\left[\left(E_{2}-B_{0}\right)\left(-i k_{2} E_{2}+B_{1}+\frac{16 C_{1}}{R_{1(2)}^{5}}\right)\right. \\
\left.+\frac{C_{0}}{R_{1(2)}^{3}}\left(4 i k_{2} E_{2}-10 B_{1}+\frac{35 C_{1}}{R_{1(2)}^{5}}\right)\right], \\
F_{2}\left(R_{1(2)}\right)= \pm 8 \chi_{\|\perp\|}\left[\left(E_{2}-B_{0}\right)\left(2 i k_{2} E_{2}-4 B_{1}+\frac{C_{1}}{R_{1(2)}^{5}}\right)\right. \\
\left.+\frac{C_{0}}{R_{1(2)}^{3}}\left(i k_{2} E_{2}-2 B_{1}-\frac{7 C_{1}}{R_{1(2)}^{5}}\right)\right] \pm 4 \chi_{b}^{(2)}\left[-\left(E_{2}-B_{0}\right)\right. \\
\left.\times \frac{10 C_{1}}{R_{1(2)}^{5}}+\frac{C_{0}}{R_{1(2)}^{3}}\left(-3 i k_{2} E_{2}+4 B_{1}-\frac{14 C_{1}}{R_{1(2)}^{5}}\right)\right], \\
F_{3}\left(R_{1(2)}\right)= \pm 12 \chi_{b}^{(2)} \frac{C_{0}^{2}}{R_{1(2)}^{7}} .
\end{gathered}
$$

Знак „,+“ соответствует $R_{1}$, знак „-“ $-R_{2}$. Коэффициенты $B_{0}, B_{1}, C_{0}, C_{1}$ определяют потенциал электрического поля внутри оболочки наночастицы [10],

$$
\begin{gathered}
B_{0}=\frac{E_{2}}{Z_{\omega}^{(0)}}\left[( \varepsilon _ { 2 } - \varepsilon _ { m } ( \omega ) ) \left(2\left(\varepsilon_{m}(\omega)-\varepsilon_{d}\right) R_{1}^{3}\right.\right. \\
\left.\left.-\left(\varepsilon_{d}+2 \varepsilon_{m}(\omega)\right) R_{2}^{3}\right)\right], \\
C_{\omega}^{(0)}=-\frac{3 \varepsilon_{2} R_{1}^{3} R_{2}^{3} E_{2}}{Z_{\omega}^{(0)}}\left[\varepsilon_{m}(\omega)-\varepsilon_{d}\right], \\
Z_{\omega}^{(0)}=\left(\varepsilon_{d}+2 \varepsilon_{m}(\omega)\right)\left(\varepsilon_{m}(\omega)+2 \varepsilon_{2}\right) R_{2}^{3} \\
+2\left(\varepsilon_{m}(\omega)-\varepsilon_{d}\right)\left(\varepsilon_{2}-\varepsilon_{m}(\omega)\right) R_{1}^{3}, \\
B_{1}=\frac{i k_{2} E_{2}}{Z_{\omega}^{(1)}}\left[( \varepsilon _ { 2 } - \varepsilon _ { m } ( \omega ) ) \left(3\left(\varepsilon_{m}(\omega)-\varepsilon_{d}\right) R_{1}^{5}\right.\right. \\
\left.\left.-\left(2 \varepsilon_{d}+3 \varepsilon_{m}(\omega)\right) R_{2}^{5}\right)\right], \\
Z_{\omega}^{(1)}=\left(2 \varepsilon_{d}+3 \varepsilon_{m}(\omega)\right)\left(2 \varepsilon_{m}(\omega)+3 \varepsilon_{2}\right) R_{2}^{5} \\
C_{1}=-6\left(\varepsilon_{m}(\omega)-\varepsilon_{d}\right)\left(\varepsilon_{2}-\varepsilon_{m}(\omega)\right) R_{1}^{5} . \\
-\frac{5 i k_{2} \varepsilon_{2} E_{2} R_{1}^{5} R_{2}^{5}}{Z_{\omega}^{(1)}}\left[\varepsilon_{m}(\omega)-\varepsilon_{d}\right], \\
\end{gathered}
$$

В этих выражениях $\varepsilon_{d}$ - диэлектрическая постоянная материала ядра частицы, $\varepsilon_{m}(\omega)$ - диэлектрическая функция материала оболочки. 
Аналогичные угловые зависимости получаются и для нормальных составляющих векторов поверхностной поляризации (5) на границах оболочки:

$$
\begin{aligned}
& P_{S \perp}^{2 \omega}\left(R_{1(2)}\right)=\left(G_{0}\left(R_{1(2)}\right)\left(3 \cos ^{2} \theta-1\right)\right. \\
& \left.+G_{1}\left(R_{1(2)}\right) \sin \theta \sin \varphi+G_{2}\left(R_{1(2)}\right) \sin ^{3} \theta \sin \varphi+G_{3}\left(R_{1(2)}\right)\right),
\end{aligned}
$$

где

$$
\begin{aligned}
G_{0}\left(R_{1(2)}\right) & = \pm \frac{\chi_{\perp \perp \perp}}{3}\left(E_{2}-B_{0}+\frac{2 C_{0}}{R_{1(2)}^{3}}\right)^{2}, \\
G_{1}\left(R_{1(2)}\right)= & \pm 2 \chi_{\perp \perp \perp}\left(E_{2}-B_{0}+\frac{2 C_{0}}{R_{1(2)}^{3}}\right) \\
& \times\left(i k_{2} R_{1(2)} E_{2}-2 B_{1} R_{1(2)}+\frac{3 C_{1}}{R_{1(2)}^{4}}\right), \\
G_{2}\left(R_{1(2)}\right)= & -G_{1}\left(R_{1(2)}\right), \quad G_{3}\left(R_{1(2)}\right)=G_{0}\left(R_{1(2)}\right) .
\end{aligned}
$$

Здесь знак „,+“ соответствует $R_{2}$, знак „-“ $-R_{1}$.

Электрическое поле удвоенной частоты можно также определить в квазистатическом приближении, решая уравнение Лапласа для сферической оболочечной частицы. На границах оболочки частицы потенциал поля испытывает скачок из-за наличия нормальной составляющей вектора поверхностной поляризации $P_{s \perp}^{2 \omega}\left(R_{1(2)}\right)$. Одновременно нормальная составляющая вектора электрической индукции претерпевает разрыв, обусловленный поверхностной плотностью заряда $\sigma_{s}^{2 \omega}\left(R_{1(2)}\right)[8,10]$. Подстановка в граничные условия слагаемых из (5) и (6) с угловой зависимостью $3 \cos ^{2} \theta-1$ приводит к результирующему полю, совпадающему с полем квадруполя с диагональным тензором квадрупольного момента, элементы которого могут быть записаны в виде

$$
\begin{gathered}
Q_{x^{\prime} x^{\prime}}^{2 \omega}=\varepsilon_{2} \alpha_{x^{\prime} x^{\prime} z^{\prime} z^{\prime}} E_{2 z^{\prime}}^{2}, Q_{y^{\prime} y^{\prime}}^{2 \omega}=\varepsilon_{2} \alpha_{y^{\prime} y^{\prime} z^{\prime} z^{\prime}} E_{2 z^{\prime}}^{2}, \\
Q_{z^{\prime} z^{\prime}}^{2 \omega}=\varepsilon_{2} \alpha_{z^{\prime} z^{\prime} z^{\prime} z^{\prime}} E_{2 z^{\prime}}^{2},
\end{gathered}
$$

где $E_{2 z^{\prime}}=E_{2}$ - амплитуда падающей на частицу волны в центре частицы, $\alpha_{x^{\prime} x^{\prime} z^{\prime} z^{\prime}}, \alpha_{y^{\prime} y^{\prime} z^{\prime} z^{\prime}}, \alpha_{z^{\prime} z^{\prime} z^{\prime} z^{\prime}}$ - компоненты тензора нелинейной квадрупольной поляризуемости наночастицы, равные

$$
\alpha_{x^{\prime} x^{\prime} z^{\prime} z^{\prime}}=\alpha_{y^{\prime} y^{\prime} z^{\prime} z^{\prime}}=-2 D_{0} /\left(3 E_{2}^{2}\right), \alpha_{z^{\prime} z^{\prime} z^{\prime} z^{\prime}}=-2 \alpha_{x^{\prime} x^{\prime} z^{\prime} z^{\prime}}
$$

где

$$
\begin{gathered}
D_{0}=\frac{4 \pi}{Z_{2 \omega}^{(0)}}\left[5\left(F_{0}\left(R_{1}\right)+2 \varepsilon_{d} G_{0}\left(R_{1}\right) / R_{1}\right) \varepsilon_{m}(2 \omega) R_{1}^{4} R_{2}^{5}\right. \\
+\left(F_{0}\left(R_{2}\right)-3 \varepsilon_{2} G_{0}\left(R_{2}\right) / R_{2}\right)\left[\left(2 \varepsilon_{d}+3 \varepsilon_{m}(2 \omega)\right) R_{2}^{5}\right. \\
\left.\left.+2\left(\varepsilon_{m}(2 \omega)-\varepsilon_{d}\right) R_{1}^{5}\right] R_{2}^{4}\right]+4 \pi G_{0}\left(R_{2}\right) R_{2}^{3} \\
Z_{2 \omega}^{(0)}=\left(2 \varepsilon_{d}+3 \varepsilon_{m}(2 \omega)\right)\left(2 \varepsilon_{m}(2 \omega)+3 \varepsilon_{2}\right) R_{2}^{5} \\
\quad+6\left(\varepsilon_{m}(2 \omega)-\varepsilon_{d}\right)\left(\varepsilon_{2}-\varepsilon_{m}(2 \omega)\right) R_{1}^{5}
\end{gathered}
$$

Положив в (7) радиус $R_{1}$ ядра частицы равным нулю, можно получить простые выражения для компонент тензора нелинейной квадрупольной поляризуемости однородной металлической наночастицы:

$$
\begin{aligned}
\alpha_{x^{\prime} x^{\prime} z^{\prime} z^{\prime}}= & \alpha_{y^{\prime} y^{\prime} z^{\prime} z^{\prime}}=-\frac{1}{2} \alpha_{z^{\prime} z^{\prime} z^{\prime} z^{\prime}}= \\
& -\frac{8 \pi R_{2}^{3}\left(2 \varepsilon_{m}(2 \omega) G_{0}\left(R_{2}\right)+R_{2} F_{0}\left(R_{2}\right)\right)}{3\left(2 \varepsilon_{m}(2 \omega)+3 \varepsilon_{2}\right) E_{2}^{2}},
\end{aligned}
$$

где

$$
\begin{gathered}
F_{0}\left(R_{2}\right)=\chi_{\|\perp\|} \frac{18 \varepsilon_{2}^{2} E_{2}^{2}}{\left(\varepsilon_{m}(\omega)+2 \varepsilon_{2}\right)^{2} R_{2}}, \\
G_{0}\left(R_{2}\right)=\chi_{\perp \perp \perp} \frac{3 \varepsilon_{2}^{2} E_{2}^{2}}{\left(\varepsilon_{m}(\omega)+2 \varepsilon_{2}\right)^{2}} .
\end{gathered}
$$

Слагаемые в (4) и (5) с угловой зависимостью $\sin \theta \sin \varphi$ являются источниками поля удвоенной частоты, совпадающего с полем диполя, дипольный момент которого направлен вдоль волнового вектора волны, падающей на частицу, и может быть представлен в виде

$$
d_{y^{\prime}}^{2 \omega}=\varepsilon_{2} \alpha_{y^{\prime} z^{\prime} z^{\prime}} i k_{2} E_{2 z^{\prime}}^{2},
$$

где $\alpha_{y^{\prime} z^{\prime} z^{\prime}}=D_{1} /\left(i k_{2} E_{2}^{2}\right)-$ единственная ненулевая компонента тензора нелинейной дипольной поляризуемости наночастицы. Для коэффициента $D_{1}$ получается следующее выражение:

$$
\begin{aligned}
D_{1}= & \frac{4 \pi}{Z_{2 \omega}^{(1)}}\left[3\left(F_{1}\left(R_{1}\right)+\varepsilon_{d} G_{1}\left(R_{1}\right) / R_{1}\right) \varepsilon_{m}(2 \omega) R_{1}^{3}\right. \\
& +\left(F_{1}\left(R_{2}\right)-2 \varepsilon_{2} G_{1}\left(R_{2}\right) / R_{2}\right)\left[\left(\varepsilon_{d}+2 \varepsilon_{m}(2 \omega)\right) R_{2}^{3}\right. \\
& \left.\left.+\left(\varepsilon_{m}(2 \omega)-\varepsilon_{d}\right) R_{1}^{3}\right]\right] R_{2}^{3}+4 \pi G_{1}\left(R_{2}\right) R_{2}^{2},
\end{aligned}
$$

где

$$
\begin{aligned}
Z_{2 \omega}^{(1)}=\left(\varepsilon_{d}\right. & \left.+2 \varepsilon_{m}(2 \omega)\right)\left(\varepsilon_{m}(2 \omega)+2 \varepsilon_{2}\right) R_{2}^{3} \\
& +2\left(\varepsilon_{m}(2 \omega)-\varepsilon_{d}\right)\left(\varepsilon_{2}-\varepsilon_{m}(2 \omega)\right) R_{1}^{3}
\end{aligned}
$$

В случае однородной металлической наночастицы компонента тензора нелинейной дипольной поляризуемости имеет простой вид

$$
\alpha_{y^{\prime} z^{\prime} z^{\prime}}=\frac{4 \pi R_{2}^{2}\left(\varepsilon_{m}(2 \omega) G_{1}\left(R_{2}\right)+R_{2} F_{1}\left(R_{2}\right)\right)}{\left(\varepsilon_{m}(2 \omega)+2 \varepsilon_{2}\right)\left(i k_{2} E_{2}^{2}\right)},
$$

где

$$
\begin{aligned}
& F_{1}\left(R_{2}\right)= \\
& =\frac{6 \varepsilon_{2}\left(-\chi_{\|\perp\|}\left(\varepsilon(\omega)-31 \varepsilon_{2}\right)+\chi_{b}^{(2)}\left(\varepsilon_{m}(\omega)+4 \varepsilon_{2}\right)\right)\left(i k_{2} E_{2}^{2}\right)}{\left(\varepsilon_{m}(\omega)+2 \varepsilon_{2}\right)\left(2 \varepsilon_{m}(\omega)+3 \varepsilon_{2}\right)}, \\
& G_{1}\left(R_{2}\right)=\chi_{\perp \perp \perp} \frac{30 \varepsilon_{2}^{2} R_{2}\left(i k_{2} E_{2}^{2}\right)}{\left(\varepsilon_{m}(\omega)+2 \varepsilon_{2}\right)\left(2 \varepsilon_{m}(\omega)+3 \varepsilon_{2}\right)} .
\end{aligned}
$$


Слагаемые в (5) и (6) с угловой зависимостью $\sin ^{3} \theta \sin \varphi$ дают вклад в поле диполя, и этот вклад можно определить, воспользовавшись мультипольным разложением потенциала от системы зарядов, распределенных по поверхностям оболочки частицы. Не зависящие от углов слагаемые в (5), (6) вклада в нелинейные дипольный и квадрупольный моменты частицы не дают.

\section{Нелинейная поверхностная поляризация границы раздела}

Следуя работе [13], нелинейную поляризацию границы раздела диэлектриков, возникающую за счет наведения в наночастицах дипольных и квадрупольных моментов, осциллирующих на удвоенной частоте, в лабораторной системе координат можно представить в виде

$$
P_{s i}=\left.\left(\chi_{i j k} E_{2 j} E_{2 k}-\chi_{i j k l} \nabla_{j} E_{2 k} E_{2 l}\right)\right|_{z=0}=P_{s i}^{2 \omega} e^{2 i\left(k_{y} y-\omega t\right)},
$$

где $\chi_{i j k}, \chi_{i j k l}$ - тензоры нелинейной дипольной и нелинейной квадрупольной поверхностной восприимчивости, $E_{2 j}-j$-я декартова компонента напряженности поля преломленной волны.

Тензоры $\chi_{i j k}$ и $\chi_{i j k l}$ связаны с тензорами нелинейных дипольной и квадрупольной поляризуемостей наночастицы правилом преобразования тензоров при поворотах системы к оординат. Для волны, поляризованной в плоскости падения, переход от системы координат $x^{\prime} y^{\prime} z^{\prime}$, связанной с наночастицей, к лабораторной системе $x y z$ (рис. 1) представляет собой поворот против часовой стрелки вокруг оси $x$ на угол, равный $\pi / 2-\varphi_{2}$, где $\varphi_{2}-$ угол преломления. Так как у тензора нелинейной дипольной поляризуемости наночастицы только одна ненулевая компонента, выражение для компонент тензора нелинейной дипольной поверхностной восприимчивости имеет простой вид

$$
\chi_{i j k}=\varepsilon_{2} n_{s} i k_{2}\left(\mathbf{e}_{i} \mathbf{e}_{y^{\prime}}\right)\left(\mathbf{e}_{j} \mathbf{e}_{z^{\prime}}\right)\left(\mathbf{e}_{k} \mathbf{e}_{z^{\prime}}\right) \alpha_{y^{\prime} z^{\prime} z^{\prime}},
$$

где круглые скобки обозначают скалярное произведение ортов и представляют собой косинусы углов между осями $y^{\prime}$ или $z^{\prime}$ исходной системы координат и осями повернутой системы, $n_{s}$ - поверхностная концентрация наночастиц.

При падении $p$-поляризованной волны у тензора нелинейной дипольной поверхностной восприимчивости будет 8 отличных от нуля компонент:

$$
\begin{gathered}
\chi_{y y y}=\varepsilon_{2} n_{s} i k_{2} \sin \varphi_{2} \cos ^{2} \varphi_{2} \alpha_{y^{\prime} z^{\prime} z^{\prime}}, \\
\chi_{y z y}=\chi_{y y z}=\varepsilon_{2} n_{s} i k_{2} \sin ^{2} \varphi_{2} \cos \varphi_{2} \alpha_{y^{\prime} z^{\prime} z^{\prime}}, \\
\chi_{y z z}=\varepsilon_{2} n_{s} i k_{2} \sin ^{3} \varphi_{2} \alpha_{y^{\prime} z^{\prime} z^{\prime}}, \\
\chi_{z y z}=\chi_{z z y}=-\varepsilon_{2} n_{s} i k_{2} \sin \varphi_{2} \cos ^{2} \varphi_{2} \alpha_{y^{\prime} z^{\prime} z^{\prime}}, \\
\chi_{z z z}=-\varepsilon_{2} n_{s} i k_{2} \sin ^{2} \varphi_{2} \cos \varphi_{2} \alpha_{y^{\prime} z^{\prime} z^{\prime}}, \\
\chi_{z y y}=-\varepsilon_{2} n_{s} i k_{2} \cos ^{3} \varphi_{2} \alpha_{y^{\prime} z^{\prime} z^{\prime}} .
\end{gathered}
$$

Компоненты тензора нелинейной квадрупольной поверхностной восприимчивости определяются формулой

$$
\begin{aligned}
\chi_{i j k l}= & \varepsilon_{2} n_{s}\left[\left(\mathbf{e}_{i} \mathbf{e}_{x^{\prime}}\right)\left(\mathbf{e}_{j} \mathbf{e}_{x^{\prime}}\right)\left(\mathbf{e}_{k} \mathbf{e}_{z^{\prime}}\right)\left(\mathbf{e}_{l} \mathbf{e}_{z^{\prime}}\right) \alpha_{x^{\prime} x^{\prime} z^{\prime} z^{\prime}}\right. \\
& +\left(\mathbf{e}_{i} \mathbf{e}_{y^{\prime}}\right)\left(\mathbf{e}_{j} \mathbf{e}_{y^{\prime}}\right)\left(\mathbf{e}_{k} \mathbf{e}_{z^{\prime}}\right)\left(\mathbf{e}_{l} \mathbf{e}_{z^{\prime}}\right) \alpha_{y^{\prime} y^{\prime} z^{\prime} z^{\prime}} \\
& +\left(\mathbf{e}_{i} \mathbf{e}_{z^{\prime}}\right)\left(\mathbf{e}_{j} \mathbf{e}_{z^{\prime}}\right)\left(\mathbf{e}_{k} \mathbf{e}_{z^{\prime}}\right)\left(\mathbf{e}_{l} \mathbf{e}_{z^{\prime}}\right) \alpha_{\left.z^{\prime} z^{\prime} z^{\prime} z^{\prime}\right]},
\end{aligned}
$$

из которой следуют выражения для 20 ненулевых компонент:

$$
\begin{aligned}
& \chi_{x x z z}=\varepsilon_{2} n_{s} \sin ^{2} \varphi_{2} \alpha_{x^{\prime} x^{\prime} z^{\prime} z^{\prime}}, \\
& \chi_{x x y y}=\varepsilon_{2} n_{s} \cos ^{2} \varphi_{2} \alpha_{x^{\prime} x^{\prime} z^{\prime} z^{\prime}}, \\
& \chi_{x x y z}=\chi_{x x z y}=\varepsilon_{2} n_{s} \sin \varphi_{2} \cos \varphi_{2} \alpha_{x^{\prime} x^{\prime} z^{\prime} z^{\prime}}, \\
& \chi_{y y y y}=\varepsilon_{2} n_{s}\left\lfloor\sin ^{2} \varphi_{2} \cos ^{2} \varphi_{2} \alpha_{y^{\prime} y^{\prime} z^{\prime} z^{\prime}}+\cos ^{4} \varphi_{2} \alpha_{z^{\prime} z^{\prime} z^{\prime} z^{\prime}}\right\rfloor, \\
& \chi_{y y y z}=\chi_{y y z y}=\varepsilon_{2} n_{s}\left\lfloor\sin ^{3} \varphi_{2} \cos \varphi_{2} \alpha_{y^{\prime} y^{\prime} z^{\prime} z^{\prime}}\right. \\
& \left.+\sin \varphi_{2} \cos ^{3} \varphi_{2} \alpha_{z^{\prime} z^{\prime} z^{\prime} z^{\prime}}\right\rfloor \\
& \chi_{y z y y}=\chi_{z y y y}=\varepsilon_{2} n_{s}\left\lfloor-\sin \varphi_{2} \cos ^{3} \varphi_{2} \alpha_{y^{\prime} y^{\prime} z^{\prime} z^{\prime}}\right. \\
& \left.+\sin \varphi_{2} \cos ^{3} \varphi_{2} \alpha_{z^{\prime} z^{\prime} z^{\prime} z^{\prime}}\right\rfloor, \\
& \chi_{y y z z}=\varepsilon_{2} n_{s}\left\lfloor\sin ^{4} \varphi_{2} \alpha_{y^{\prime} y^{\prime} z^{\prime} z^{\prime}}+\sin ^{2} \varphi_{2} \cos ^{2} \varphi_{2} \alpha_{z^{\prime} z^{\prime} z^{\prime} z^{\prime}}\right\rfloor, \\
& \chi_{z z y y}=\varepsilon_{2} n_{s}\left\lfloor\cos ^{4} \varphi_{2} \alpha_{y^{\prime} y^{\prime} z^{\prime} z^{\prime}}+\sin ^{2} \varphi_{2} \cos ^{2} \varphi_{2} \alpha_{z^{\prime} z^{\prime} z^{\prime} z^{\prime}}\right\rfloor \text {, } \\
& \chi_{y z y z}=\chi_{z y z y}=\chi_{z y y z}=\chi_{y z z y}= \\
& \varepsilon_{2} n_{s}\left\lfloor-\sin ^{2} \varphi_{2} \cos ^{2} \varphi_{2} \alpha_{y^{\prime} y^{\prime} z^{\prime} z^{\prime}}+\sin ^{2} \varphi_{2} \cos ^{2} \varphi_{2} \alpha_{z^{\prime} z^{\prime} z^{\prime} z^{\prime}}\right\rfloor, \\
& \chi_{z z z z}=\varepsilon_{2} n_{s}\left\lfloor\sin ^{2} \varphi_{2} \cos ^{2} \varphi_{2} \alpha_{y^{\prime} y^{\prime} z^{\prime} z^{\prime}}+\sin ^{4} \varphi_{2} \alpha_{\left.z^{\prime} z^{\prime} z^{\prime} z^{\prime}\right\rfloor},\right. \\
& \chi_{z z z y}=\chi_{z z y z}=\varepsilon_{2} n_{s}\left\lfloor\sin \varphi_{2} \cos ^{2} \varphi_{2} \alpha_{y^{\prime} y^{\prime} z^{\prime} z^{\prime}}\right. \\
& \left.+\sin ^{3} \varphi_{2} \cos \varphi_{2} \alpha_{z^{\prime} z^{\prime} z^{\prime} z^{\prime}}\right\rfloor, \\
& \chi_{z y z z}=\chi_{y z z z}=\varepsilon_{2} n_{s}\left\lfloor-\sin ^{3} \varphi_{2} \cos \varphi_{2} \alpha_{y^{\prime} y^{\prime} z^{\prime} z^{\prime}}\right. \\
& \left.+\sin ^{3} \varphi_{2} \cos \varphi_{2} \alpha_{z^{\prime} z^{\prime} z^{\prime} z^{\prime}}\right\rfloor .
\end{aligned}
$$

Таким образом, вектор нелинейной поверхностной поляризации будет иметь две составляющие:

$$
\begin{aligned}
& P_{s y}^{2 \omega}=\chi_{y y y} E_{2 y}^{2}+\left(\chi_{y z y}+\chi_{y y z}\right) E_{2 y} E_{2 z}+\chi_{y z z} E_{2 z}^{2} \\
& -2 i k_{y}\left(\chi_{y y y y} E_{2 y}^{2}+\left(\chi_{y y z y}+\chi_{y y y z}\right) E_{2 y} E_{2 z}+\chi_{y y z z} E_{2 z}^{2}\right) \\
& +2 i k_{2 z}\left(\chi_{y z y y} E_{2 y}^{2}+\left(\chi_{y z y z}+\chi_{y z z y}\right) E_{2 y} E_{2 z}+\chi_{y z z z} E_{2 z}^{2}\right), \\
& P_{s z}^{2 \omega}=\chi_{z y y} E_{2 y}^{2}+\left(\chi_{z y z}+\chi_{z z y}\right) E_{2 y} E_{2 z}+\chi_{z z z} E_{2 z}^{2} \\
& -2 i k_{y}\left(\chi_{z y y y} E_{2 y}^{2}+\left(\chi_{z y z y}+\chi_{z y y z}\right) E_{2 y} E_{2 z}+\chi_{z y z z} E_{2 z}^{2}\right) \\
& +2 i k_{2 z}\left(\chi_{z z y y} E_{2 y}^{2}+\left(\chi_{z z y z}+\chi_{z z z y}\right) E_{2 y} E_{2 z}+\chi_{z z z z} E_{2 z}^{2}\right),
\end{aligned}
$$

где $E_{2 y}$ и $E_{2 z}-y$ - и $z$-проекции амплитуды преломленной $p$-волны,

$$
E_{2}=\frac{2 \sqrt{\varepsilon_{1}} \cos \varphi_{0}}{\sqrt{\varepsilon_{1}} \cos \varphi_{2}+\sqrt{\varepsilon_{2}} \cos \varphi_{0}} E_{0}
$$


$E_{0}-$ амплитуда падающей волны, $\varphi_{0}-$ угол падения.

Для волны, поляризованной перпендикулярно плоскости падения (s-волны), переход от $x^{\prime} y^{\prime} z^{\prime}$ к $x y z$ представляет собой два последовательных поворота против часовой стрелки: сначала поворот вокруг оси $z^{\prime}$ на угол, равный $\pi / 2-\varphi_{2}$, затем вокруг оси $y$ на угол $3 \pi / 2$. В результате у тензора нелинейной дипольной поверхностной восприимчивости будет 2 отличные от нуля компоненты

$$
\begin{gathered}
\chi_{z x x}=-\varepsilon_{2} n_{s} \cos \varphi_{2} \alpha_{y^{\prime} z^{\prime} z^{\prime}} \\
\chi_{y x x}=\varepsilon_{2} n_{s} \sin \varphi_{2} \alpha_{y^{\prime} z^{\prime} z^{\prime}}
\end{gathered}
$$

Ненулевых компонент тензора нелинейной квадрупольной поверхностной восприимчивости также три:

$$
\begin{aligned}
& \chi_{x x x x}=\varepsilon_{2} n_{s} \alpha_{z^{\prime} z^{\prime} z^{\prime} z^{\prime}}, \\
& \chi_{z z x x}=\varepsilon_{2} n_{s} \alpha_{x^{\prime} x^{\prime} z^{\prime} z^{\prime}}, \\
& \chi_{y y x x}=\varepsilon_{2} n_{s} \alpha_{y^{\prime} y^{\prime} z^{\prime} z^{\prime}} .
\end{aligned}
$$

В результате вектор нелинейной поверхностной поляризации также будет иметь $y$ - и $z$-компоненты

$$
\begin{aligned}
& P_{s y}^{2 \omega}=\left(\chi_{y x x}-2 i k_{y} \chi_{y y x x}\right) E_{2 x}^{2}, \\
& P_{s z}^{2 \omega}=\left(\chi_{z x x}+2 i k_{2 z} \chi_{z z x x}\right) E_{2 x}^{2},
\end{aligned}
$$

где

$$
E_{2 x}=\frac{2 \sqrt{\varepsilon_{1}} \cos \varphi_{0}}{\sqrt{\varepsilon_{1}} \cos \varphi_{0}+\sqrt{\varepsilon_{2}} \cos \varphi_{2}} E_{0},
$$

- амплитуда преломленной $s$-волны.

Нелинейная поверхностная поляризация, определяемая формулами (11), (12) для падающей $p$-волны и (13), (14) для $s$-волны, является источником плоских волн с частотой $2 \omega[15]$, уходящих от границы раздела сред. Эти волны поляризованы в плоскости падения. Согласно работе [15], амплитуда отраженной ВГ гармоники равна

$$
E^{2 \omega}=\frac{4 \pi i k_{1}^{s}}{\varepsilon_{1} k_{2 z}^{s}+\varepsilon_{2} k_{1 z}^{s}}\left(k_{2 z}^{s} P_{s y}^{2 \omega}-k_{y}^{s} P_{s z}^{2 \omega}\right),
$$

где $k_{1}^{s}=2 \omega \sqrt{\varepsilon_{1}} / c-$ волновое число в верхнем диэлектрике, $c$ - скорость света в вакууме,

$$
\begin{gathered}
k_{y}^{s}=2(\omega / c) \sqrt{\varepsilon_{1}} \sin \varphi_{0}, \\
k_{1 z}^{s}=\sqrt{(2 \omega / c)^{2} \varepsilon_{1}-\left(k_{y}^{s}\right)^{2}}, \\
k_{2 z}^{s}=\sqrt{(2 \omega / c)^{2} \varepsilon_{2}-\left(k_{y}^{s}\right)^{2}}
\end{gathered}
$$

- компоненты волнового вектора ВГ в верхнем и нижнем диэлектриках. Интенсивность ВГ пропорциональна квадрату модуля амплитуды (15).

\section{Результаты расчетов и выводы}

При проведении расчетов для описания частотной зависимости диэлектрической проницаемости материала оболочки частицы использовалась обобщенная модель Друде

$$
\varepsilon_{m}(\omega)=\varepsilon_{\infty}-\omega_{p l}^{2} /\left(\omega^{2}+i \gamma \omega\right),
$$

где $\varepsilon_{\infty}-$ высокочастотная диэлектрическая проницаемость, учитывающая вклад ионной решетки, $\omega_{p l}$ и $\gamma$ - плазменная частота и коэффициент диссипации, определяющий тепловые потери в металле. Характеристики благородного металла выбирались соответствующими серебру: $\hbar \omega_{p l}=9.1 \mathrm{eV}, \hbar \gamma=0.027 \mathrm{eV}$, $\varepsilon_{\infty}=4.45$ [18]. Диэлектрическая проницаемость $\varepsilon_{d}$ ядра частицы варьировала от 2 до 3. Диэлектрические постоянные оптически прозрачных сред принимались равными $\varepsilon_{1}=1, \varepsilon_{2}=2$. Внешний радиус частицы $R_{2}$ составлял $5 \mathrm{~nm}$. Радиус ядра частицы изменялся от 0 до $3 \mathrm{~nm}$. Напряженность поля в падающей волне была равна $3 \cdot 10^{6} \mathrm{~V} / \mathrm{cm}$. Поверхностная концентрация наночастиц составляла $10^{11} \mathrm{~cm}^{-2}$. Выражения для компонент тензора нелинейной поверхностной восприимчивости и объемной восприимчивости металла были взяты из работы [17]:

$$
\begin{aligned}
\chi_{\perp \perp \perp} & =\frac{a e}{16 \pi m \omega^{2}}\left(\varepsilon_{m}(\omega)-1\right), \\
\chi_{\|\perp\|} & =\frac{b e}{16 \pi m \omega^{2}}\left(\varepsilon_{m}(\omega)-1\right), \\
\chi_{b}^{(2)} & =\frac{d e}{16 \pi m \omega^{2}}\left(\varepsilon_{m}(\omega)-1\right),
\end{aligned}
$$

где $m$ и $e-$ масса и заряд электрона, $a, b, d-$ коэффициенты Рудника и Штерна. В рамках гидродинамической модели для этих коэффициентов получаются следующие значения: $a=1, b=-1, d=1[19,20]$. Однако сравнение результатов экспериментальных исследований ГВГ от металлических пленок с теорией показало, что коэффициенты Рудника и Штерна отличаются от предсказываемых теоретически. В данной работе при проведении расчетов использовались значения из [17]: $a=7, b=-0.3, d=0.07$.

Результаты расчетов относительной интенсивности $I^{2 \omega} / I_{0}\left(I^{2 \omega} \sim\left|E^{2 \omega}\right|^{2}, I_{0} \sim\left|E_{0}\right|^{2}\right)$ отраженной волны удвоенной частоты в зависимости от длины падающей электромагнитной волны $p$-поляризации представлены на рис. 2 и 3.

На рис. 2 приведены спектральные зависимости относительной интенсивности $I^{2 \omega} / I_{0}$ ВГ при разных радиуcax $R_{1}$ ядра частицы. Диэлектрическая постоянная $\varepsilon_{d}$ ядра частицы равнялась 2 . Угол падения $\varphi_{0}$ составлял $50^{\circ}$. Сплошная кривая 1 на рис. 2, a соответствует однородной серебряной частице $\left(R_{1}=0\right)$. В диапазоне длин падающей волны от 700 до $1000 \mathrm{~nm}$ на спектральной зависимости наблюдаются два пика. Правый пик отвечает дипольному плазмонному резонансу в наночастицах на удвоенной частоте, который наблюдается при выполнении равенства $\operatorname{Re}\left[\varepsilon_{m}(4 \pi c / \lambda)\right]=-2 \varepsilon_{2}$, что следует из 

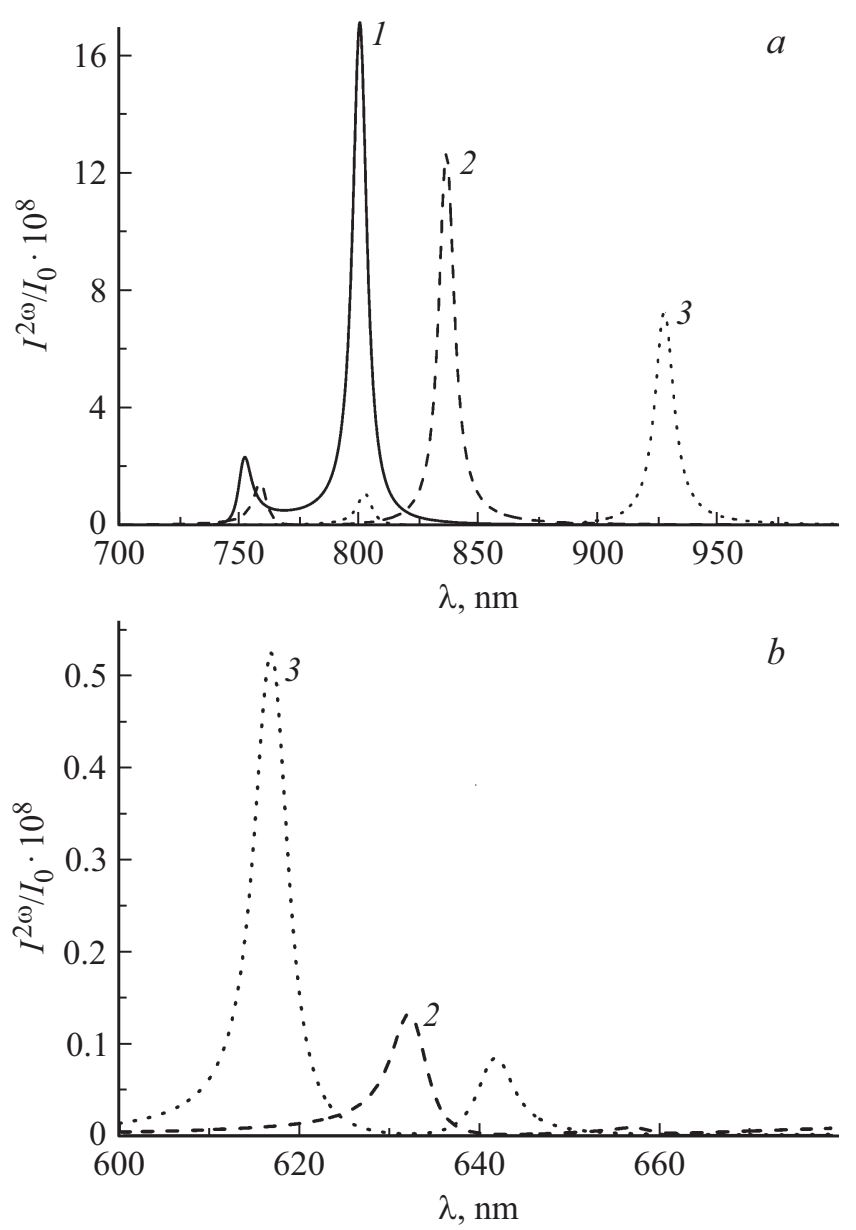

Pис. 2. Спектральные зависимости относительной интенсивности ВГ при разных радиусах ядра частицы: $R_{1}=0(1)$, 2 (2), $3 \mathrm{~nm}$ (3) в диапазонах длин волн 700-1000 (a) и $600-700 \mathrm{~nm}(b)$.

формулы (10). Левый пик обусловлен квадрупольным плазмонным резонансом, и его положение определяется равенством $\operatorname{Re}\left[\varepsilon_{m}(4 \pi c / \lambda)\right]=-3 \varepsilon_{2} / 2$, как видно из формулы (8). Появление у частицы диэлектрического ядра приводит к смещению этих пиков в сторону больших длин волн и к уменьшению их высоты - штриховая (2) и пунктирная (3) кривые на рис. 2, $a$. Кроме того, в диапазоне длин волн от 600 до $700 \mathrm{~nm}$ появляются слабые пики, обусловленные наличием внутренней границы ,диэлектрик-металл““, как видно из рис. $2, b$. В этой спектральной области правый пик соответствует квадрупольному, а левый пик - дипольному плазмонным резонансам на удвоенной частоте в наночастицах. При увеличении ядра частицы эти пики смещаются в сторону меньших длин волн, и их высота увеличивается. Положения пиков кривых 2 и 3 на рис. 2 определяются минимумами знаменателей $Z_{2 \omega}^{(0)}$ и $Z_{2 \omega}^{(1)}$ в формулах (7) и (9).

На рис. 3 изображены спектральные зависимости при разных значениях диэлектрической постоянной $\varepsilon_{d}$ ядра частицы. Сплошная кривая 1 соответствует $\varepsilon_{d}=2$, штриховая 2-2.5, пунктирная 3-3. Радиус $R_{1}$ ядра частицы составлял $2 \mathrm{~nm}$. Угол падения $\varphi_{0}$ был равен $50^{\circ}$. Как видно из рисунка, увеличение $\varepsilon_{d}$ приводит к смещению резонансов ВГ в сторону больших длин волн. При этом высота пиков, обусловленных плазмонным резонансом на внешней границе оболочки, уменьшается (рис. $3, a$ ), а пиков, обусловленных наличием внутренней границы оболочки, увеличивается (рис. $3, b$ ).

Как показывают расчеты, зависимость $I^{2 \omega} / I_{0}$ от угла падения электромагнитной волны является гладкой. $I^{2 \omega} / I_{0}$ возрастает от нуля при $\varphi_{0}=0^{\circ}$, достигает максимума при $\varphi_{0} \approx 60^{\circ}$, а затем убывает до нуля при $\varphi_{0}=90^{\circ}$.

При падении волны $s$-поляризации зависимости $I^{2 \omega} / I_{0}$ от длины падающей волны при разных радиусах и диэлектрических проницаемостях ядра частицы аналогичны рассмотренному выше случаю волны $p$-поляризации. Имеются только незначительные количественные отличия.

Таким образом, проведенное в настоящей работе теоретическое исследование показало, что спектральное положение максимумов ВГ, генерируемой монослоем плаз-
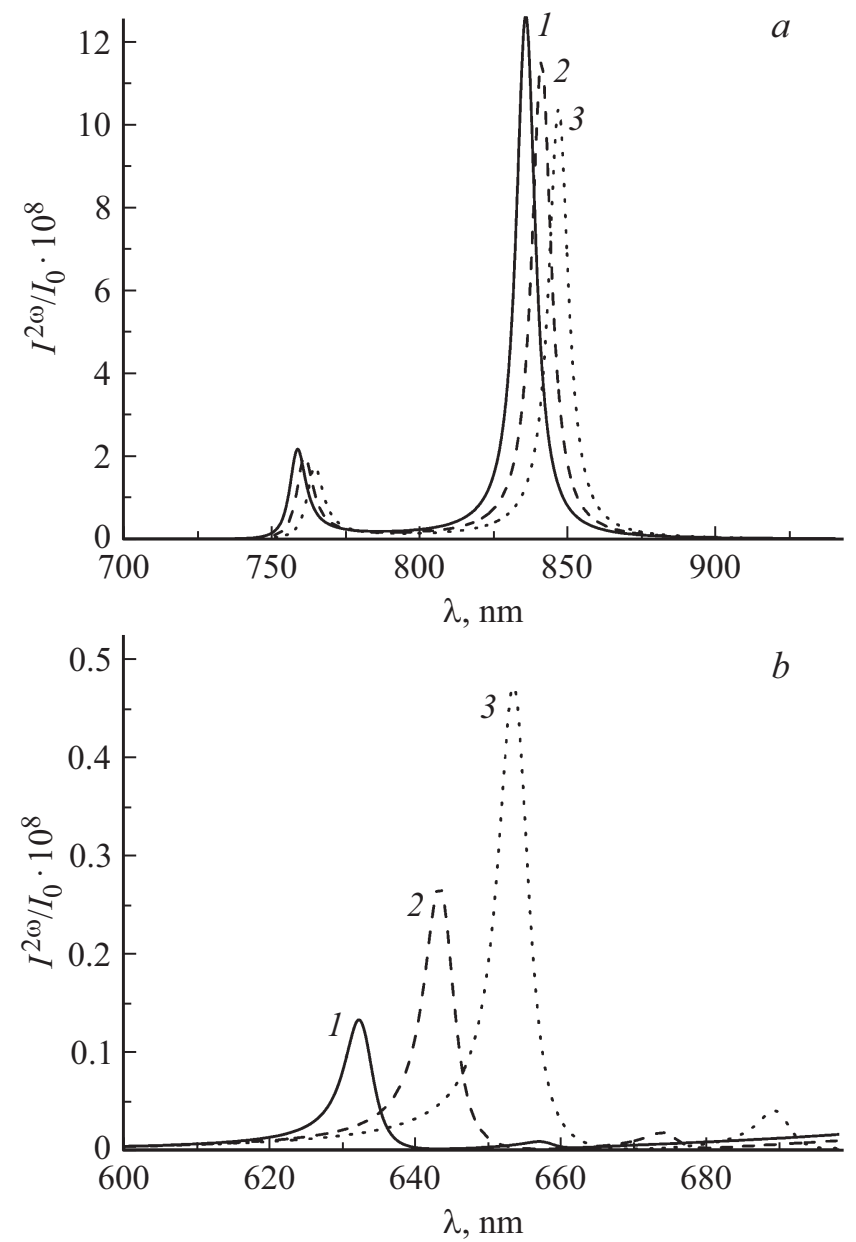

Рис. 3. Спектральные зависимости относительной интенсивности ВГ при разных диэлектрических постоянных ядра частицы: $\varepsilon_{d}=2(1), 2.5(2), 3(3)$ в диапазонах длин волн $700-900(a)$ и $600-700 \mathrm{~nm}(b)$. 
монных наночастиц, расположенным на плоской границе раздела двух оптически прозрачных сред, существенным образом зависит от диэлектрической проницаемости ядра частицы и его размера, а также от угла падения возбуждающей волны. Варьируя эти характеристики, можно добиваться интенсивной ГВГ в нужной спектральной области. Это может иметь важное прикладное значение, например, при разработке новых источников электромагнитного излучения, в конструкцию которых входят ансамбли двухслойных плазмонных наночастиц.

\section{Финансирование работы}

Исследование выполнено при поддержке Министерства науки и высшего образования Российской Федерации, проект № FSGU-2020-0003.

\section{Конфликт интересов}

Авторы заявляют, что у них нет конфликта интересов.

\section{Список литературы}

[1] Bachelier G., Butet J., Russier-Antoine I., Jonin C., Benichou E., Brevet P.F. // Phys. Rev. B. 2010. V. 82. P. 235403. doi 10.1103/PhysRevB.82.235403

[2] Malvezzi A.M., Allione M., Patrini M., Stella A., Cheyssac P., Kofman R. // Phys. Rev. Lett. 2002. V. 89. P. 087401. doi 10.1103/PhysRevLett.89.087401

[3] Ferrara D., McMahon M.D., Lopez R., Haglund R.F. // Proc. SPIE. 2007. V. 6458. P. 64581K. doi 10.1117/12.708293

[4] Czaplicki R., Husu H., Siikanen R., Mäkitalo J., Kauranen M., Laukkanen J., Lehtolahti J., Kuittinen M. // Phys. Rev. Lett. 2013. V. 110. P. 093902. doi. 10.1103/PhysRevLett.110.093902

[5] Czaplicki R., Kiviniemi A., Huttunen M.J., Zang X., Stolt T., Vartiainen I., Butet J., Kuittinen M., Martin O.J.F., Kauranen M. // Nano Lett. 2018. V. 18. P. 7709. doi 10.1021/acs.nanolett.8b03378

[6] Smirnova D., Kivshar Yu.S. // Optical Society of America. Optica. 2016. V. 3. N 11. P. 1241. doi 10.1364/OPTICA.3.001241

[7] De Beer A.G.F., Roke S. // Phys. Rev. B. 2008. V. 79. P. 155420. doi 10.1103/PhysRevB.79.155420

[8] Brudny V.L., Mendoza B.S., Mochan W.L. // Phys. Rev. B. 2000. V. 62. P. 11152. doi 10.1103/PhysRevB.62.11152

[9] Ильин Н.В., Смирнова Д.А., Смирнов А.И. // Вестник ННГУ. Радиофизика. 2013. Т. 6. С. 74.

[10] Чмерева Т.М., Кучеренко М.Г. // Изв. вузов. Физика. 2021. T. 64. № 1. C. 145; Chmereva T.M., Kucherenko M.G. // Russian Phys. J. 2021. V. 64. N 1. P. 168. doi 10.1007/s11182-021-02313-6

[11] Forestiere C., Capretti A., Miano G. // J. Opt. Soc. Am. B. 2013. V. 30. N 9. P. 2355. doi 10.1364/JOSAB.30.002355

[12] Zhang L., Fan Z.H., Chen R. // Optics Express. 2017. V. 25. N 23. P. 28010. doi 10.1364/OE.25.028010

[13] Jerome B., Shen Y.R. // Phys. Rev. E. 1993. V. 48. N 6. P. 4556. doi 10.1103/PhysRevE.48.4556

[14] Feller M.B., Chen W., Shen Y.R. // Phys. Rev. A. 1991. V. 43. N 12. P. 6778. doi 10.1103/PhysRevA.43.6778
[15] Shen Y.R. // Annu. Rev. Phys. Chem. 1989. V. 40. P. 327. doi 10.1146/annurev.pc.40.100189.001551

[16] Dadap J.I., Shan J., Yeinz T.F. // J. Opt. Soc. Am. B. 2004. V. 21. N 7. P. 001328. doi 10.1364/JOSAB.21.001328

[17] Krause D., Teplin C.W., Rogers C.T. // J. Appl. Phys. 2004. V. 96. N 7. P. 3626. doi $10.1063 / 1.1786341$

[18] Климов В.В. Наноплазмоника. М.: Физматлит, 2009. 480 с.

[19] Sipe J.E., So V.C.Y., Fukui M., Stegeman G.I. // Phys. Rev. B. 1980. V. 21. N 10. P. 4389. doi 10.1103/PhysRevB.21.4389

[20] Rudnick J., Stern E.A. // Phys. Rev. B. 1971. V. 4. N 12. P. 4274. doi 10.1103/PhysRevB.4.4274 